\title{
Enhancing the Performance of a High-Gain \\ Free Electron Laser Operating \\ at Millimeter Wavelengths
}

W. A. Barletta, B. Anderson, W. N. Fawley, V. K. Nei1, T. J. Orzechowski, D. Prosnitz, E. T. Scharlemann and S. M. Yarema (LLNL) A. C. Paul, D. Hopkins, A. M. Sessler and J. Wurtele (LBL)

This paper was prepared for subiaittal to The liorkshop on Coherent ano Collective Properties of Relativistic E-beams and

Electromagnetic Radiation

Villa $01 \mathrm{mo}$, Como, Italy September 13-16, 1984

October 25, 1984

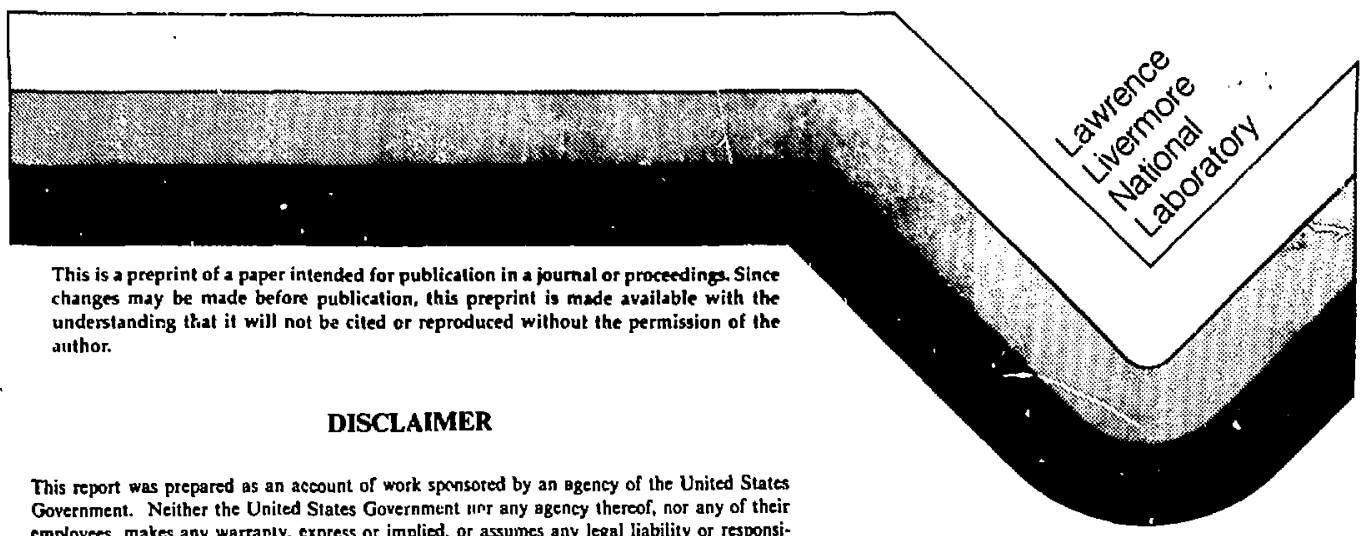
employees, makes any warranly, express or implied, or assumes any legal liability or responsibijity for the acsuracy, completeness, or usefulness of any information, apparatus, product, or process disciused, or represents that its use would not infringe privately owned rights. Reference herein to any specilic commercial product, process, or service by trade anme, trademart, manufacturer, or otherwise does not necessarily constitute or imply its endorsement, recommendation, or favoring by the United States Government or any agency thereof. The views and opinions of authors expressed herein do not necessarily state or eflect those of the United States Government or any agency thercof.

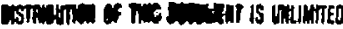

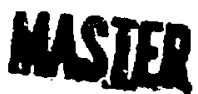


ENHANCING THE PERFORMANCE OF A HIGH-GAIN FREE ELECTRON LASER OPERATING AT MIL'.IMETER WAVELENGTHS

\section{W. A. Barletta, B. Anderson, W. M. Fawley, V. K. Neil, \\ T. J. Orzechowski, D. Prosnitz, E. r. Scharlemann and S. M. Yarema Lawrence Livermore National Laboratory* \\ Livermore, California 94550 U.S.A.} A. C. Paul, D. Hopk ins, /A. M. Sessler and J. Wurtele
Lawrence Berkeley Laboratory+
Berkeley. Cal ifornio 94720 U.S.A.

\section{ABSTRACT}

A high-gain, high extraction efficiancy, free electron laser (FEL) amplifier ${ }^{1}$ operating at the Experimental Test Acceierator (ETA) at $34.6 \mathrm{GHz}$ has demonstrated a small signal gain of $13.4 \mathrm{~dB} / \mathrm{m}$. With a $30 \mathrm{~kW}$ input signal, the amplifier has produced a saturated output of $80 \mathrm{MW}$ and a $5 \%$ extraction efficiency. Comparison of these results with a linear model ${ }^{2}$ at small signal levels indicates that the amplifier can deliver saturatea output starting from noise, if the brightness of the electron beam is sufficiently high. The brightness of the ETA is far below that possible with optimized choice of practical design characteristics such as peak voltage, cathode type, gun electrode geometry, and focusing field topology. In particular, the measured brightness of the ETA injector is limited by plasma effects ${ }^{3}$ from the present cold, plasma cathode. As part of a coordinated theoretical and experimental effort to improve injector performance, we are using the $E B Q^{4}$ gun design code to explore the current limits of gridiess, relativistic, Pierce colunins with moderate current density $\left(>50 \mathrm{~A} / \mathrm{cm}^{2}\right)$ at the catliode. The chief component in our experimental effort is a readily modified electron gun that will allow us to test many candidate cathode materials, types, and electrode geometries at field stresses up to $1 \mathrm{MV} / \mathrm{cm}$.

\footnotetext{
ॠ Performed jointly under the auspices of the U.S. DOE by LLNL under $W-7405-E N G-48$ and for the DOD under DARPA, ARPA Order No. 4395, monitored by NSWC.

+ This work was supported by the Director, Advanced Energy Systems, Basic Energy Sciences, Office of Energy Pesearch, US DOE under contract number DE-ACO3-76SF00098.
} 


\section{MOTIVATION}

The Electron Laser Facility (ELF) is designed to be a test of the physical models used to predict high-gain and high-efficiency FEL operation in the visible spectral region. ELF consists of an anplifier with well-defined initial conditions on the radiation and the electron beam and with no axial magnetic field. The Lawrence Livermore Hational Laboratory's (LLNL) ETA provides a $6 \mathrm{kA}, 3.3 \mathrm{MeV}$ beam with a normalized emittance of 1.5 , radian-cm to the ELF. An emittance filter reduces the beam current to approximateiy v0o A with a normalized edge emittance* of .47 * radian-cm. Another $100 \mathrm{~A}$ of current are lost in the quadrupole transport line used to match the beam into the $3 \mathrm{~m}$ long wiggler.

Measurements ${ }^{5}$ of the signal gain in the superradiant mode (no microwave input signal) shown in Fig 1 , indicate that the microwave signal grew at a rate of $13.4 \mathrm{~dB} /$ meter for a beam current of $450 \mathrm{~A}$. Extrapolating this growth back to the origin, one finds an effective input noise of $0.35 \mathrm{~W}$. Near the magnetic field strength corresponding to the peak output of a 1-meter long wiggler, examination of the amplifier gain as a function of wiggler length (Fig. 2) with a $30 \mathrm{~kW}$ input signal clearly reveals that the ampiifier goes into saturation at 2.2 meters. Beyond this point, the amplified cutput power first decreases and then near 3 meters starts to increase again. The gain as a function of wiggler length shows an exponential growth of approximately 15.6 dB/meter up to saturation $\left(L_{w}=2.2 \mathrm{~m}\right)$. This value is in close agreement with the small signal gain measurement.

*Edge emittance is defined as the area in phase space which matches the acceptance of the emittance selector and contains $100 \%$ of the electron beam. 
The linear theory ${ }^{2}$ best suited to describe the experiment has been derived by linearizing the single particle, 10 ditudinal $(y-\psi)$ equations of motion derived by Kroll, Morton and Rosenbluth. ${ }^{6}$ The procedure is identical to that in Ref. 7, with the addition of explicit betatron motion and an integration over the waveguide. This version of the linear theory predicts the very steep dependence of gain on the electron beam radius shown in Fig.... 3. The observed exponential gain, after accounting for fractional coupling into the growing mode (launching losses), corresponds to a maximum beam radius of approximate 1 y $8 \mathrm{~mm}$.

$8 y$ increasing the brightness of the electron beam by an order of magnitude above its present value of $10^{4} \mathrm{~A} / \mathrm{cm}^{2}-\mathrm{rad}^{2}$, we expect that the maximum betatron radius $c$ an be reduced to less than $0.4 \mathrm{~nm}$ with more than $500 \mathrm{~A}$ transported through ihe wiggler. The linear theory predicts that the small signal gain will thereby increase to $>40 \mathrm{~dB} / \mathrm{m}$. As two gain is likely to be limited to about one e-folding per wiggler period $(9.8 \mathrm{~cm})$, ten e-foldings per meter is probably the maximum gain achievable with the present ELF wiggler. Nonetheless, we expect gains high enough that the noise-generated output of ihe first meter of ELF will exceed the signal needed to drive the remaining two-meters of wiggler into saturation.

Even in the absence of tapering the wiggler, reducing the emittance of the intense beam driving the ELF amplifier can result in a source of tunable miliimeter waves of hundreds of megawatts. The extremely high gain possible from very bright, multi-kiloampere electron beams is especialiy important for amplifiers of wavelengths in the one to several millimeter range, in which sources with strengths exceeding several kilowatts are rare. As described below, our initial theoretical and experimental investigations into the limits 
of beam brightness indicate that values exceeding $10^{6} \mathrm{~A} / \mathrm{cm}^{2}-\mathrm{rad}^{2}$ should be attainable.

\section{I. SOURCES OF BEAM EMITTANCE}

The brightness of a given beam source is set by a variety of practical design choices such as peak voltage, cathode type, gun electrode geometry, and focusing field topology: From" a more fundamental puint of view, we can identify several physical effects which can conlribute to the emittance $\varepsilon$ in a relativistic electron gun. These effects include: (1) source temperaturf, (2) source uniformity, (3) magnetic field normal to the cathode, (4) beam filamentation, (5) non-linear applied forces, (6) nor-linear space charge forces, and (7) multiplicity or motion of emissive surfaces.

1. Source Temperature (T). The normalized source brightness, $B$, is related to the temperature $T(e V)$ and sourre emissivity $\mathrm{J}\left(A / \mathrm{cm}^{2}\right)$ by

$$
B=\frac{j m(e V)}{T T(e v)}=1.6 \times 10^{5} \mathrm{~J} / T .
$$

In existing high current guns, this contribution is insignificant in comparison to other contributors to emittance. However, as we push brightnesses toward $10^{6} \mathrm{~A} / \mathrm{cm}^{2}-\mathrm{rad}^{2}$, maintaining non-emission limited sources with an effective temperature below 1 eV will be critical for cathode types with enissivity less than $10 \mathrm{~A} / \mathrm{cm}^{2}$.

2. Source Uniformity. To the extent that emission is non-uniform, the beam transport through regions dominated by non-linear radial forces will entrain "phase space vacuum" via phase mixing. As this dilution can become irreversible after one-quarter of a betatron wavelength, an estimate of this effect is obtained by using as the emittance volume of the smallest ellipse 
enclosing all significant current regions at the cathode surface. Maintaining source uniformity argues in favor of small area cathodes wich high emissivity.

3. Normal Magnetic Field. In the presence of a magnetic field normal to the cathode surface, electrons are emitted with a finite canonical angular momentum $P_{\theta}$. When the electrons leave the region of axial field,. . .. they acquire a kinetic momentum sufficient to keep their canonical angular momentum constant. This motion gives the beam an equivalent emittance in both transverse planes: $\quad \varepsilon=e B_{2} R^{2} / 2 y a m c$ where $B_{2}$ is the mean normal field, $R$ is the cathode radius; $r s$ are the usual relativistic factors; $m$ is the electron mass; and $c$ is the speed of light. Reducing the cathode radius reduces this contribution in two was: (1) tire emittance scales as $R^{2}$, and (2) the average value of $B_{2}$ can be kept mare near zero over a smaller area. 4. Filamentaicion by a Grid. In gun designs with a grid, each hole in the grid can act as a focusing or defocusing lens with a focal length given by

$$
1 / f=\epsilon(\Delta E) / 2 \gamma B^{2} m c^{2}
$$

where $\Delta E$ is the voltage difference across the grid.

The phase space is distorted by the finite grid spacing, occurring via filamentation downstream of the grid. Paul and Neil have described the effect $^{2}$ in the ETA gun; they report the effect to be exceeded by the effects of non-linear forces. In the design of extremely bright, high current guns, however, grid filamentation may be a limiting factor unless the grid material is extremely fine and the grid highly transparent. Unfortunately, such a grid is unlikely to be compatible with hot thermionic or dispenser cathodes, which have a low effective electron temperature. 
Moreover, this incompatibility will be exacerbated if the gun is operated at a repetition rate exceeding a few tens of Hertz. Moving the grid to the anode plane will minimize thermal loading and beam filamentation, but can lead to catastrophic over-focusing of multi-kiloampere beams. The most prudent choice in the design of a high brightness gun is to seek a design in which the grid has been omitted.

5. Non-linear Applied Forces. The radial forces from tire applied electric and magnetic fields in guns such as ETA and ATA injectars have significant anharmonic components (proportional to $R^{3}$ ). In general, these effects can be made to compensate for each other by careful design with a simulation code such as EBQ. In such simulations one must account for the other significant source of anharmonic fields, the non-linear self-fields of the beam itself.

6. Non-linear Space Charge Forces. The strong space-charge forces of an intense, low energy beam will distort the free space equipotentials to result in a defocussing spherical aberation in the beam transport. Proper shaping of the potentials by a graded accelerating column with shaped electrodes (Pierce correction) can eliminate this effect for a particular operating condition; that is, for a specified operating voltage and beam current.

Self-forces can also lead to increase in emittance whenever the beam cannot be matched into the transport. At high currents beam loading of the gun's drive circuits will lead to an energy variation correlated with time variation of the beam current. As the initial transport is space charge dominated, not all segments of the beam can be matched into the transport downstream of the injector. Phase mix damping of the mis-match osciflations will lead to an increase of beam emittance. ${ }^{8}$ 
7. Emission from Positions of Different Potentials. Even if the electrons are born with zero intrinsic temperature, the beam can acquire significant emittance in the extraction process if the electrons originate on different equipotentials. Such multiple source beams have an instantaneous energy spread which will phase-mix into macroscopic emittance as the beam is accelerated and transported through the space charge dominated regime. The same consideration will limit the pulse length over which an ideal pierce correction can be applied, if the cathode surface is a moving piasma sheath. Extraction from under-dense, moving plasmas are a worst case example of multiple, equipotential emission. Thr cold, surface flashover cathode used in the ETA for the ELF experiments produces such a piasma.

An important tool for evaluating the relative contribution of the several sources of beam enittance is a computational model of relativistic electron beam dynamics, which can accommodate the vide range of realistic electrode geometries possible to the designer. For this purpose, LLNL developed the EBO simulation code, which was used for the design of the $10 \mathrm{kA} A T A$ gun. EBD uses a steady-state, self-consistent calculation of the particle trajectories associated with high current relativistic beam propugation in axially symmetric injectors possessing external, two-dimensional electric and magnetic fields. The code treats the coherent electric and magnetic self-fields of the beam with sufficient accuracy in the relativistic regime, to model the high degree of cancellation which occurs between the self-magnetic and self-electric furces. 


\section{APPROACHES TO HIGH BRIGHTNESS}

Our efforts to push the brightness of multi-kiloampere electron beams to the practical limits are proceeding along three fronts: cathode improvement, simulation and test of gridless designs, and study of transient phenomena. Candidate cathode types and injector designs are tested in the ETA and in readily modified injector High Brightness Test Stand (HBTS).

\section{A. CATHODE TYPES}

Our survey of cathode types is limited to soures which can deliver $5 \mathrm{kA}$ at $>10 \mathrm{~Hz}$ with emissivity, $J>5 \mathrm{~A} / \mathrm{cm}^{2}$. The categories we have identified appear in Table 1. We wish to avoide cathodes that require vacuums bettef than $10^{-8}$ Torr, that are easily poisoned, or that have operating lives $<500$ hours. We consider maintaining source uniformity over areas exceesing a few hundred $\mathrm{cm}^{2}$ impractical. Unless accelerating flelds are extremely high $(>1 \mathrm{MV} / \mathrm{cm})$ control of beam space charge in high $\mathrm{J}\left(>500 \mathrm{~A} / \mathrm{cm}^{2}\right)$ designs will be difficult; hence, the source brightness in laser heated cathodes can be lost immediately. Uncontrolled field emisson sources are attractive for initial experiments. Presentiy controlled field emitters may be useful for sources with brightness up to $10^{6}$; however, these sources are still experimental and unavailable for early experiments. Controlled porosity, dispenser cathodes seem to offer the greatest promise for reliable operation at the limits of brightness.

\section{B. DESIGN STUDIES}

In our numerical simalations we have yet to find a multi-kiloampere injector design with a brightness limited by the cathode brightness. Gun 
optics and control of beam space charge dominate the emittance. Once one has chosen an extended cathode $\left(>100 \mathrm{~cm}^{2}\right)$, the preferred path seems to be to find an approximation to a gridless, relativistic Pierce column. The requirement in a Pierce column is that the electrons experience no net radial force. The difficulty with this approach is well known from the design of non-relativistic Pierce columns: the external field to null the radial forces applies to a single operating condition: beam current, voltage and cathode location; moreover, even this "point" solution requires a large number of intermediate electrodes to grade and shape the field.

A cursory analytic examination reveals the characteristics and the difficulties of this approach. It is well known that for a relativistic beam the forces from the space charge repulsion, $\varepsilon_{r s}$, and the magnetic pinch fieid cancel to within a factor of $\gamma^{-2}$. To balance the total radial self-force the applied electric field, $E_{r a}$, is

$$
E_{r a}=-E_{r s} / r^{2}
$$

For a beant with charge density, $\rho$, the total radial electric field, $E_{r}$, will cancel the magnetlc pinch force, if

$$
E_{r}=2 \pi \rho \beta^{2} r
$$

Combining (2) with Gauss's law, we can write a differential equation for the accelerating field, $E_{2}$ :

$$
\frac{\partial E_{2}}{\partial z}=\frac{4 \pi}{\gamma^{2}} \rho \quad-4 \pi \beta p \frac{\partial B}{\partial r} .
$$


The kinetic energy equation relates $\gamma$ to the accelerating field;

$$
\frac{\partial r}{\partial z}=\frac{e}{m c^{2}} \quad E_{z}
$$

Inserting (4) into (3) and expressing the charge density in terms of the current density, $J$, we have the defining equation for the Pierce solution:

$$
\frac{\partial 2}{\partial z^{2}}=\frac{4 \pi J}{B \gamma}\left(\frac{e}{m c}\right)-4 \pi J\left(\frac{e}{m c^{3}}\right) \frac{\partial B}{\partial r} \cdot
$$

Neglecting the second term in (5), we can integrate the equation to obtain the solution for $y$ and thus the equipotentials in the gun as $a$ function of $z$ displayed in Fig. 4. The figure displays the characteristic bunching of the equipotentials as the beam approaches the anode. Satisfying this relationship is sufficient for low current, relativistic electron guns.

Turning our attention to the second term in Eq. (5), we see that it is negligible when the potential depression is small, that its contribution increases faster than linearly with beam current, and that: it is most important when $Y$ is near one; i.e., near the cathode. Qualitatively, this term forbids an exact solution for all $r$ and all $z$ from a planar cathode; if the forces are balanced at the center of the beam the outer edge will be overfocused. Conversely, if the forces are balanced at the edge of the beam the center will be underfocused. Fortunately, however, the contribution of this term can be minimized by shaping the cathode so that the initial trajectories of the edge rays of the beam are diverging. Balancing the forces on center can then lead to a condition in which all rays are nearly parallel once the beam energy is high enough that the space charge depression contributes an insignificant amount to the electron velocity. 
An idealization of this appruach is illustrated in Fig. 5; the electron source is a flat cathode plate surrounded by an annular shroud. Field shaping is accomplished by two artifacts: (1) a sharp anode tip bunches the equipotentials as requied in an ideal Pierce column, and (2) the surface connecting the anode tip normal to the shroud is a Neumann boundary, forcing the tangential electric field to vanish along the surface. The cathude has a slightly convex sirape. Steady-state culculations with $E B Q$ of wie electron trajectories and the macroscopic brightness as a function of axial position in the gun are given in Figs. 5 (b), (c), respectively. The current limits of this approach as well as its practical realization are under investigation for experimental test on HBTS. Reiaxation of either of the two characteristic features of the design degraues the brightness by un order of magnitude. Moreover, traisient effects may alter these results for sirart pulses.

As a code benchmark we note that EQB code simulations of the ASTKON gun reveal that the dominant contribution to emittance was the relativistic magnetic self pinch on approach to the anode foil. The calculated emittance scated to the operating condtions of 500 Amperes at $6 \mathrm{meV}$ is $0.019 \mathrm{~cm}-\mathrm{mrad}$; the measured vaiue was in the range of $0.01-0.03 \mathrm{~cm}-\mathrm{mrad}$. The ETA gun operating at $8 \mathrm{kA}$ has a calculated emittance of $0.6 \mathrm{~cm}$-rad giving a brightness of a few times $10^{4}$. This value compares favorably with observed performance. 


\section{EXPERIMENTAL STUDIES}

Central to our experimental studies a reidily modified $2 \mathrm{MV}$, several $\mathrm{KA}$ electron gun HBTS that will allow us to test candidate cathode materials, types and electrode geometries. The HBTS is designed to sccomnodate electron sources ranging from small field emission cathodes requiring up to $1 \mathrm{MV} / \mathrm{cm}$ fields to medium area $\left(<100 \mathrm{~cm}^{2}\right)$ cathodes such as thermionic or plasma sources with moderate extraction fields $(-100 \mathrm{iV} / \mathrm{cm})$. The stand allows evaluation of not only ine intrinsic brightness of the cathode but diso the source ruggedness, usefulness for high average power operation and lifetime.

The power drives for the HBTS are MAG-1, magnetic madulators are capable of more than $100 \mathrm{~Hz}$ continuous operation. The radiation shieiding is a pool clesigned to provide $10^{5}$ attenuation. A 15 hp pump combined with a $10^{4}$ galion storage tank provides for a 15 minute removal of the radiation shielding. The two halvas of the injector are independently mounted on tracks in order to facilitate access to the interior for work on the electrodes. A beam transport extending into a second underground roon will provide an area where a sample of the electron beam can be analyzed.

\section{v. CONCLUSIONS}

The ELF amplifier should be able to produce saturated output generated from noise if we can ihicrease beam brightness to $10^{5} \mathrm{~A} / \mathrm{cm}^{2}-\mathrm{rad}^{2}$. - We find no practical or theoretical reasons why the brightness of multi-kilnampere guns such as the ETA injector cannot be pushed well above $10^{6} \mathrm{~A} / \mathrm{cm}^{2}-\mathrm{rad}^{2}$. Cathodes presently under development show promise for providing rugged, reliable beam sources of kiloampere beams at brightness as high as $10^{7}$. At such high brightness the trade-offs between beam brightness and total current must be re-evaluated. 


\section{REFERENCES}

1. T. J. Orzechowski et al., "Free Electron Generators of Coherent Radiation," C. A. Brau, S. F. Jacobs, M. O. Scully, Editors (SPIE, Bellingham, WA, ) p. 65 (1983).

2. E. T. Scharleman and A. M. Sessler, "The Linear Theory of the Microwave Free Electron Laser at ELF." Report in preparation.

3. W. A. Barletta, J. K. Boyd, D. S. Prono, A. C. Paul, "Brightness Limitations in Multi-kiloampere Electron Beam Sources," LLNI UCRL-90914 (September 1984 ).

4. A. C. Paul, "The EBQ Code," LBL-13241 (Novenber \$982).

5. T. J. Orzechowski et al, Microwave Radiation from a High-Gain Free Electron Laser Amplifier," submitted to Phys. Rev. Letters (September 1984).

6. N. M. Kroll, P. L. Morton and M. R. Rosenbluth, IEEE J. Quantum Electronics QE-17, p. 1436 (1981).

7. R. Bonifacio, C. Pelligrini and L. M. Narducci, Brookhaven National Laboratory Report BNL-34340 (1983).

8. F. P. Lee, S. S. Yu and W. A. Barletta, "Phase-Space Distortion of a Heavy Ion Beam Propagating through a Vacuum Reactor Vessel," Nucl. Fusion Vol. 21, No. $\underline{8}(1981)$. 


\section{FIGURE CAPTIONS}

FIG. I Small signal gain in the super-radiant mode as a function of wiggler length.

FIG. 2 Microwave power output as a function of wiggier length for constant wiggler field $\left(B_{W}=4280\right.$ Gausss).

FIG. 3 Small signal gain as derived from linear theory as a function of maximum betatron radius of the beam electrons.

FIG. 4 Relativistic Pierce solution neglecting the effect of space charge depression on eleciron velocity.

FIG. 5 Conceptual design of a high brightness, multi-kiloamp electron gun. 


\begin{tabular}{|c|c|c|c|c|c|c|c|c|}
\hline \multicolumn{2}{|c|}{ Cathode type } & $T_{\text {eff }}(e V)$ & $J\left(A / \mathrm{cm}^{2}\right)$ & $\mathrm{B}\left(\mathrm{A} / \mathrm{ra \textrm {G } ^ { 2 }}-\mathrm{cm}^{2}\right)$ & Area $\left(\mathrm{cm}^{2}\right)$ & Vacuum (Torr) & Lifetime (hr) & Comments \\
\hline Thermionic & $\mathrm{BaO}_{6}$ & $\begin{array}{l}\sim 0.1 \\
\sim 0.2\end{array}$ & $\begin{array}{l}\sim 5 \\
\leqslant 30\end{array}$ & $\begin{array}{r}510^{7} \\
<3 \times 10^{7} \\
\end{array}$ & $\begin{array}{r}-1000 \\
-\quad 200\end{array}$ & $\begin{array}{l}10^{-7} \\
10^{-7}\end{array}$ & $\begin{array}{l}<10^{3} \\
<10^{3} \\
\end{array}$ & $\begin{array}{l}\text { Moderately susceptible } \\
\text { to poisoning } \\
\text { High operating temperature }\end{array}$ \\
\hline Dispenser & $\begin{array}{l}\text { Random } \\
\text { porosity } \\
\text { Controlled } \\
\text { porosity }\end{array}$ & $\begin{array}{l}\sim 0.2 \\
\sim 0.2\end{array}$ & $\begin{array}{l}<30 \\
\leqslant 40\end{array}$ & $\begin{array}{l}<3 \times 10^{7} \\
\leqslant 5 \times 10^{\prime}\end{array}$ & $\begin{array}{l}-200 \\
-200\end{array}$ & $<5 \times: r^{-7}$ & $10^{3}$ & $\begin{array}{l}\text { Uniformity depends } \\
\text { on porosity } \\
\text { Uniform, rugged }\end{array}$ \\
\hline Laser heated & & $\geqq 0.2$ & $\sim 10^{3}$ & $-3 \times 10^{8}$ & -5 & $10^{-8}$ & $10^{3}$ & $\begin{array}{l}\text { Easily poisoned, } \\
\text { not mature }\end{array}$ \\
\hline Photo-emissive & & $<0.1$ & - & - & - & $\sim 10^{-11}$ & $\sim 10^{3}$ & \\
\hline Field emission & $\begin{array}{l}\text { Controlled } \\
\text { Uncontrolled }\end{array}$ & 1 & $\begin{array}{c}600 \\
100-1000\end{array}$ & $\begin{array}{l}2 \times 10^{8} \\
3 \times 10^{7}\end{array}$ & $\begin{array}{c}-10 \\
-5-50\end{array}$ & $10^{-6}$ & $>10^{3}$ & $\begin{array}{l}\text { Experimental, rugged } \\
\text { Rugged }\end{array}$ \\
\hline lashboard & & $\sim 70$ & $\sim 30$ & $\sim 10^{5}$ & $\sim 200$ & $10^{-6}$ & $>10^{4}$ & $\begin{array}{l}\text { Multi-component } \\
\text { beam }\end{array}$ \\
\hline
\end{tabular}

Table 1 


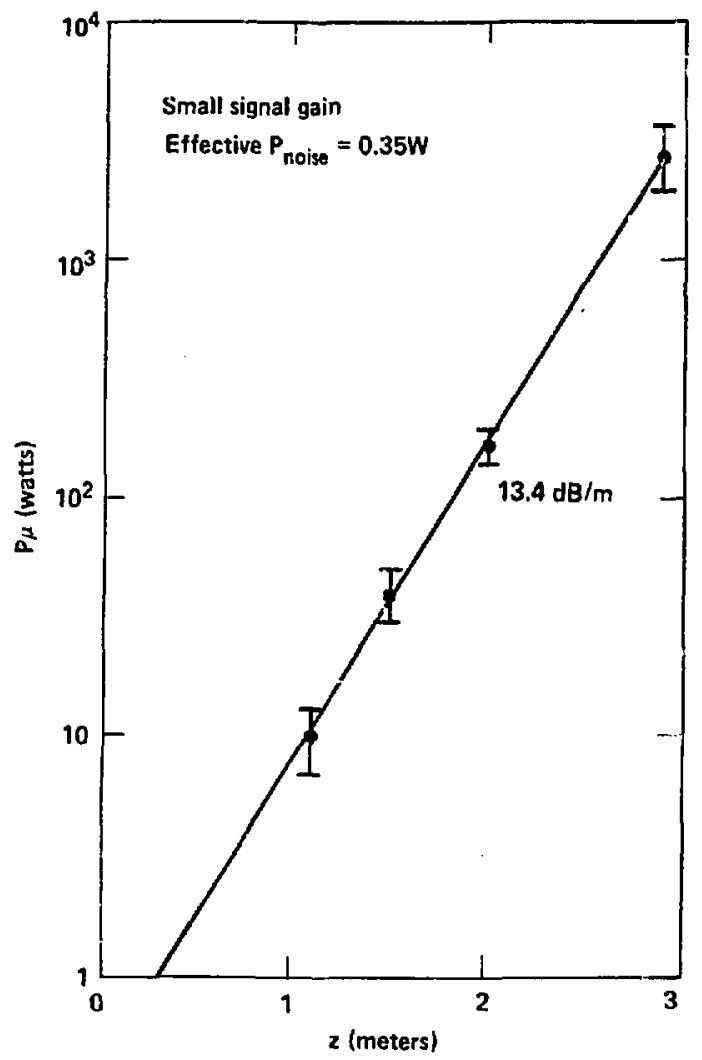

Fig. 1 


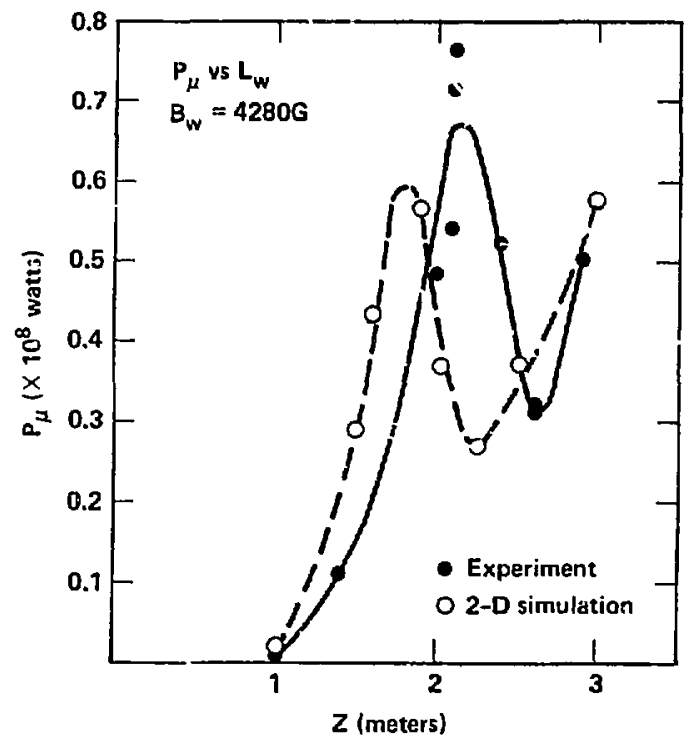

Fig. 2 


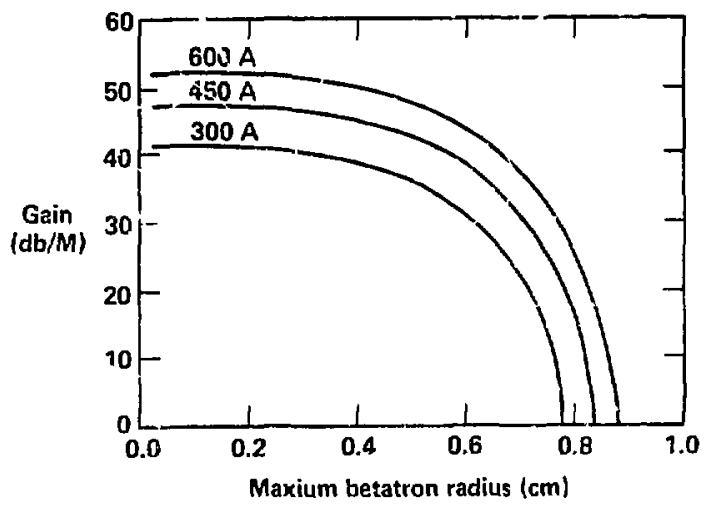

Fig. 3 


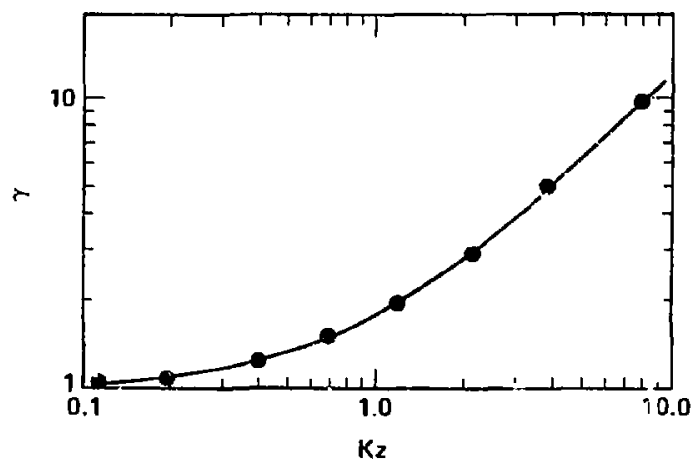

Fig. 4

$-19-$ 

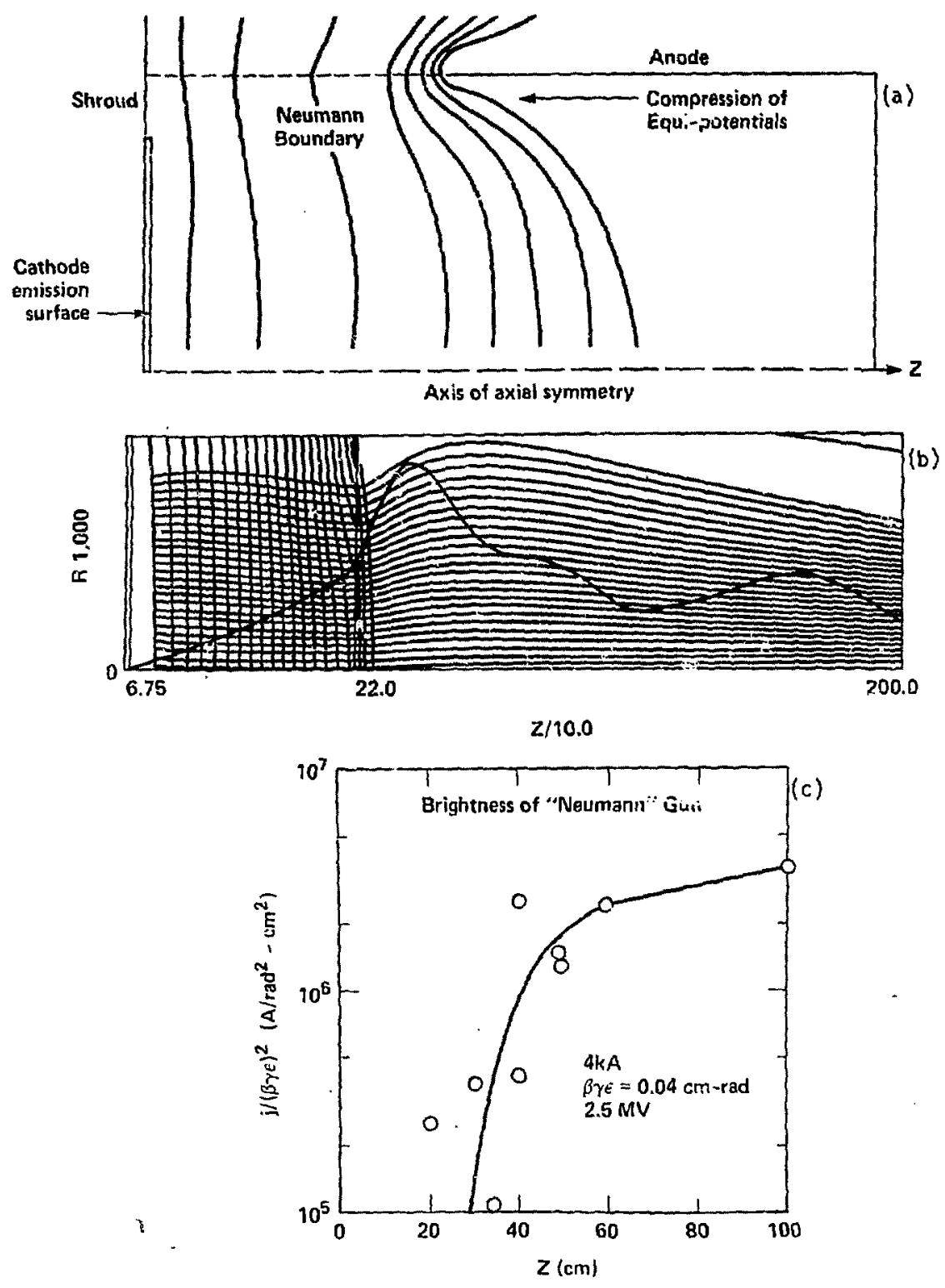

Fig. 5 